\title{
Hydroxypropylation of high-amylose maize starch changes digestion and fermentation-dependent parameters in rats - ERRATUM
}

Kiyoshi Ebihara, Makoto Tachibe, Natsumi Kaneko and Taro Kishida

\section{Development of the gut microbiota in southern Indian infants from birth to 6 months: a molecular analysis - ERRATUM}

Jayakanthan Kabeerdoss, Shama Ferdous, Ramadass Balamurugan, John Mechenro, R. Vidya, Sridhar Santhanam, Atanu K. Jana and Balakrishnan S. Ramakrishna

Journal of Nutritional Science (2013), vol. 2, e19, page 1

doi:10.1017/jns.2013.20

The publishers regret to announce that the papers by Ebihara $e t$ al. ${ }^{(1)}$ and Kabeerdoss $e t$ al. ${ }^{(2)}$ were published with the incorrect volume and article numbers. The correct volume and citation details are as follows:

Ebihara K, Tachibe M, Kaneko N, et al. (2013) Hydroxypropylation of high-amylose maize starch changes digestion and fermentation-dependent parameters in rats. J Nutr Sci 2, e17 doi:10.1017/jns.2013.5.

Kabeerdoss J, Ferdous S, Balamurugan R, et al. (2013) Development of the gut microbiota in southern Indian infants from birth to 6 months: a molecular analysis. J Nutr Sci 2, e18 doi:10.1017/jns.2013.6.

The publisher sincerely apologises for this error.

\section{References}

1. Ebihara K, Tachibe M, Kaneko N, et al. (2013) Hydroxypropylation of high-amylose maize starch changes digestion and fermentation-dependent parameters in rats. J Nutr Sci 2, e17 doi:10.1017/jns.2013.5.

2. Kabeerdoss J, Ferdous S, Balamurugan R, et al. (2013) Development of the gut microbiota in southern Indian infants from birth to 6 months: a molecular analysis. J Nutr Sci 2, e18 doi:10.1017/jns.2013.6. 